\title{
Typhoid fever: hurdles to adequate hand washing for disease prevention among the population of a peri-urban informal settlement in Fiji
}

\author{
James Greenwell, a Judith McCool,b Jacob Koolc and Mosese Salusalud \\ Correspondence to James Greenwell (e-mail: i.greenwell@auckland.ac.nz).
}

Problem/context: The Pacific island nation of Fiji Islands has high rates of endemic typhoid fever which is difficult to diagnose and often underreported. However, the majority of cases are preventable through use of safe water; adequate sanitation; vaccination; and, most sustainable of all, simple hygienic behaviour, such as hand washing with soap (HWWS). Despite many attempts by public health authorities, little progress has been made in the area of environmental adaptation and behaviour change.

Action: To explore perceptions of typhoid fever risk among urban squatters and behavioural determinants surrounding HWWS, indigenous Fijians living in informal settlements with high typhoid fever incidence were invited to participate in focus group discussions. In-depth interviews were conducted with community leaders.

Outcome: Perceptions of typhoid fever suggest confusion about risk factors, symptoms and communicability. Environmental barriers for hand washing were related to water and soap access. Standard social marketing approaches have been trialled with little clear evidence of impact. Despite this, we continue to advocate for the social and cultural determinants of typhoid prevention to remain central to future public health strategies.

Discussion: Despite behaviour change being notoriously difficult, we argue that community-driven behaviour adaptation initiatives based on sound epidemiological evidence and health communication theory are likely to have significant impact and greater likelihood of sustainability.

$\mathrm{T}$ yphoid is endemic in Fiji as indicated by the 1847 laboratory-confirmed cases reported between January 2008 and July $2012 .{ }^{1}$ To put this figure in context, the population of Fiji is approximately 840000 . The rate of typhoid fever has increased in recent years, likely due to a combination of surveillance improvements and rapid urbanization. In the past decade the poverty rate also rose from $25 \%$ to $50 \%$ of the population. As the poverty rate has increased, many Fijians have moved from rural areas to informal squatter settlements in urban centres where the majority have little access to clean water and sewerage infrastructure. ${ }^{2}$

Age and gender remain important to the epidemiology of typhoid in Fiji; the mean age of typhoid fever patients was 27 years, and $57 \%$ of cases were male $(1043 / 1847)$. For both males and females, the age group with the heaviest burden of disease was 20-29 year olds. ${ }^{3}$ A combination of untreated waste; a proliferation of bore hole wells for washing and consumption; frequent flooding; and inadequate water, sanitation and hygiene infrastructure in the informal squatter settlements are associated with increased risk of typhoid fever. ${ }^{2}$ The majority of typhoid fever cases (93\%) that presented to hospitals in 2008 were ethnic Fijians, and more than half of those cases were males in the $20-30$ and $30-39$ age ranges. ${ }^{4}$

\section{CONTEXT}

Previous research suggests that typhoid prevention and control require a multifaceted approach incorporating sanitation, low-cost hand hygiene and hand washing with soap interventions alongside vaccination programmes. ${ }^{5,6}$

\footnotetext{
Social and Community Health, School of Population Health, University of Auckland, Auckland, New Zealand.

Global Health, School of Population Health, University of Auckland, Auckland, New Zealand.

Division of Pacific Technical Support, World Health Organisation, Suva, Fiji.

Fiji School of Medicine, Fiji National University, Fiji.

Submitted: 9 October 2012; Published: 10 January 2013

doi: 10.5365/wpsar.2012.3.4.006
} 
Evidence from international studies indicates that between $30 \%$ and $45 \%$ of gastro-intestinal illnesses and a further $30 \%$ of acute respiratory illnesses can be simply prevented by adequate hand washing with soap at key moments: before handling food and after defecation. ${ }^{7-9}$ Understanding the motivating factors for routine hand washing is essential to any initiative likely to result in sustained population behaviour change. ${ }^{6,10}$ We conducted a qualitative study among residents of a urban settlement in Suva, Fiji, to (1) explore how people living in an informal settlement perceived the relevance and impact of hand washing to prevent typhoid, and (2) explore how the risk of typhoid is prioritized alongside other competing health and social challenges.

\section{ACTION}

A qualitative study design, using in-depth interviews and focus groups, was used to develop a detailed understanding of factors that influence habitual hand washing as a preventive measure against typhoid. ${ }^{11}$

The focus group discussion data was collected from a semi-urban settlement of around 400 people located near the capital city of Suva. The informal (i.e. urban slum or squatter) settlement is situated on the edge of the densely populated Suva-Nausori corridor. The settlement was established in the 1960s and received piped municipal water several hours per day to a few households and to a stand pipe following a typhoid fever outbreak in the early 2000 s.

There were four focus groups (two male groups and two female groups) each with up to seven participants. The groups were divided by age roughly based on groups identified by a 2010 typhoid fever study from the Northern Division in Fiji. ${ }^{4}$ The age groups were young males/females (aged 18-29) and older males/females (aged 30 and above). A sample of 27 participants was recruited for this study; the majority were indigenous Fijians and included a Methodist pastor from the settlement, a community prayer leader/community organizer, two local health centre nurses and a local behaviour change expert from a Suva-based nongovernmental organization. There were a total of five "thought-leaders" (e.g. church or women's group leaders) interview participants.

Focus group discussions and in-depth interviews were carried out in and around the informal settlement during March of 2011. Discussions involving residents of a peri-urban informal settlement were conducted in Fijian by local undergraduate Fiji National University public health students who also transcribed and translated the discussion into English. The focus group discussions were imported into NVivo Version 9 to assist with data management for thematic analysis. ${ }^{12}$

\section{OUTCOME}

The following key findings from the group discussions and interviews were developed out of a thematic analysis of the interview data. Substantiating quotes from the members of the group with minimal identifier information gender (male $[\mathrm{M}]$, female $[\mathrm{F}]$ ) and group number based on age (18-29 years old [1], 30 years old and above [2]) are provided.

\section{Confusion and ambivalence}

For the majority of participants, perceptions about typhoid risk factors were obtained from a recent mass media prevention campaign. Typhoid prevention posters were used as decoration on the wall of the house where the interviews took place. Pamphlets, radio and television advertisements also promoted the risk of typhoid; however, attention on the disease rather than the common risk factors, such as poor hand washing efficacy, confused the focus for some participants.

“...when I came home from work there was a pamphlet explaining typhoid, describing how it is spread and ways of preventing it; I also saw information on the TV. I am confused between typhoid and filariasis due to the information on posters in the hospitals and the pamphlets." (1F)

Contradictions or misinterpretation of public health messages was evident. Despite many participants being able to recite public health messages, experiential knowledge and behaviours were not consistent.

"The important message received from ads is washing hands after using the toilet and before meals. It is important for people to wash their hands to stop the spread not only of typhoid but also some other diseases. Sometimes I don't wash my hands because 
I do not believe that I would get the disease, but other times I do wash my hands." (2F)

“...seeing the poster has brought about fear that typhoid is here and it is real; that fear encourages me to practise proper hygiene. Sometimes I don't wash my hands because I do not believe that I would get the diseases." (2M)

By far, the most significant driver to behaviour change was first-hand experience with disease in the local community.

"The community got really sick and they got scared and that was the factor when everyone started to pay attention. If there is a case around then we get really serious and start to worry." (1F)

“...don't want my family to go through what I did, so I encourage them, especially the children, to wash their hands." (2F)

\section{Brand name soap - the ideal soap?}

Female participants expressed preference for the widely promoted soap, Protex ${ }^{\circledR}$. There was a pervasive belief that soaps were ranked in quality, and those that are promoted widely (on billboards) are most desired, even if not used. Indeed, the pervasive commercial advertising for more expensive soap brands was well recognized among female participants. The marketing and resulting perceptions among the participants contradicts the official hand washing media which advocate the use of more affordable laundry soap. Successful marketing of expensive commercial soap brands has led to the incorrect perception that expensive antibacterial soaps are required to prevent disease.

"Sometimes people think it's a waste to use on the hands...it is not the only soap that kills germs... not everyone can afford Protex ${ }^{\circledR}$ soap. People think that hand washing with Protex ${ }^{\circledR}$ is the only way to prevent typhoid fever." (1F)

Emotional drivers to hand washing included disgust, protection and nurturance. Other affective motivators such as worry and fear about unwanted attention from landlords and health authorities (after a typhoid case was notified) were also evident.

“...She doesn't want her family to go through what she did, and therefore she encourages hand washing in her family, but sometimes she forgets... (2F)

"Remove visible dirt when it is not greasy and dirty. Just don't touch anything dirty. Wash with a good soap. Some soap you wash your hands with may not make your hands clean." (1M)

\section{Community driven programmes - a key determinant of behaviour change?}

Hand washing interventions were notably more salient when conducted by members of the local community. Ad hoc initiatives, presented by outsiders were undermined and largely were disregarded.

"...we talked about it once, but when the health workers leave things will go back to normal." (1F)

“...Information directly from community members would make a difference, and people would actually act on the advice given because they have seen first-hand information about typhoid and not just heard or seen it on radio and television. Information given by those who have actually had typhoid is important. The ads on TV are good, but they're not as effective as community members going from house to house to inform people." (1M)

Bathing and laundry were the primary means of hand washing (by proxy) rather than deliberate hand washing with soap at the key junctures, such as when preparing food or eating meals. Laundry and bathing the body are so intrinsic to basic hygiene and are closely motivated by the need to nurture and or feel clean. Oral hygiene was also widely reported as habitual.

"Washing dishes and showering is one way we wash hands. Doing the laundry and washing the dishes are opportunities to wash hands." (2F) 
"...back at home at 6pm to have shower, wash properly then have dinner and off to bed...Wake up, brush teeth and wash face, sometimes bathe..." (2M)

\section{Water - a pervasive environmental challenge}

Some households in the settlement still rely on well water; however, access to piped water has improved for many in the settlement since 2007. A fundamental and pervasive appreciation of the value of water was notable among participants. Clean water was a scarce commodity (particularly water that had not been preused or stagnant).

Poor access to water (due mainly to service cuts and shortages with piped water infrastructure) and soap combined with a belief that hand washing required large amounts of water undermined any behavioural intention. Indeed, laundry and bathing were considered higher priorities than hand washing.

“...can't waste so much water just for washing hands, since there is shortage of water; it's time consuming to use well water during water cuts. The hands are just a small part of the body." (1F)

“...People use basins to wash dishes, but they might use the same water to wash hands because they don't want to waste water." (2F)

\section{DISCUSSION}

Our findings reveal several key areas for potential improvement. First, confusion persists over transmission of and effective protection from typhoid fever within this settlement. Access to clean and reliable water remains a barrier; the reliance on stored water as backup for daily use is a disincentive for consistent hand washing with clean water. In addition, the mass marketing of antibacterial soaps has undermined the perceived efficacy of ordinary, low cost soaps for hand washing. Finally, social marketing efforts, such as posters and pamphlets are attractive and collectable, but appear to have minimal impact in providing a consistent and salient message that may, alongside other environmental changes, effect change.

Health communications addressing environmental adaptation behaviours (necessitated by contextual challenges) and that also provide feedback on the costs and benefits of hand washing behaviour might prove more effective than those that provide standard health education messages or hygiene techniques. Environmental barriers to hand washing, such as access to clean water and soap, were a significant but not exclusive deterrent to hand washing. These factors exist in the context of general ambivalence about the benefits of timely hand washing and perceptions of clean versus dirty hands. Common beliefs about bacterial transmission and risks to family health were somewhat erratic, based on prior experience and family routines and habits but not on formal knowledge or instruction. Health communications could also emphasize that relatively small amounts of water and soap can be effective if hand washing with soap is performed at minimum key junctures. Similarly, social barriers for hand washing with soap such as traditional gender and age (elder) related status influence behaviour and norm-setting in communities. Focusing on establishing behavioural norms that are responsive to the environment (that is, higher risk and lower resources) may produce longer-term intrinsic changes among that population.

\section{CONCLUSION}

Behaviour change is the single most challenging dimension of public health. Low efficacy health education efforts are testament to the public health challenges. ${ }^{13-15}$ The added challenge for promoting hand washing is that the consequences are often delayed. Drawing upon the intrinsic values within a community such as looking good to others, protecting children and family, smelling good and not feeling dirty might have greater currency than objective instructional messages. ${ }^{6,14,15}$ Either way, community-driven initiatives based on sound epidemiological evidence and health communication theory are likely to have significant impact and greater likelihood of sustainability beyond the life of the intervention. 


\section{Conflicts of interest}

None declared.

\section{Funding}

None.

\section{References:}

1. Scobie $\mathrm{H}$ et al. Preliminary Report: Impact Assessment of the 2010 Mass Typhoid Vaccination Campaign, Republic of Fiji. Suva, Centers for Disease Control and Prevention, World Health Organization, Fiji Ministry of Health, Australian Agency for International Development, 2011.

2. World Health Organization. Meeting Report: Expert Consultation on Typhoid Fever Vaccination in Fiji. Suva, Fiji Ministry of Health, 2010.

3. Jenkins K. Post Cyclone Tomas Support to Typhoid fever control in Fiji March 2010. Suva, Fiji Health Sector Improvement Program, 2010.

4. Alefaio $L$ et al. Typhoid Fever Outbreaks in the Northern Division of Fiji Islands, 2008. Informaction, 2009, 30:4.

5. Samuelsen $\mathrm{H}$ et al. Hygiene and sanitation among ethnic minorities in Northern Vietnam: Does government promotion match community priorities? Social Science \& Medicine, 2010.

6. Curtis VA, Danquah LO, Aunger RV. Planned, motivated and habitual hygiene behaviour: an eleven country review. Health Education Research, 2009, 24:655-673. doi:10.1093/her/ cyp002 pmid:19286894
7. Xue Y. Hand washing for preventing diarrhoea. Journal of Advanced Nursing, 2008, 64:14-15. doi:10.1111/j.13652648.2008.04783.x

8. Allegranzi $B$ et al. Religion and culture: potential undercurrents influencing hand hygiene promotion in health care. American Journal of Infection Control, 2009, 37:28-34. doi:10.1016/j. ajic.2008.01.014 pmid:18834738

9. Aiello $A E$ et al. Effect of hand hygiene on infectious disease risk in the community setting: a meta-analysis. American Journal of Public Health, 2008, 98:1372-1381. doi:10.2105/ AJPH.2007.124610 pmid:18556606

10. Whitby $\mathrm{M}$ et al. Behavioural considerations for hand hygiene practices: the basic building blocks. The Journal of Hospital Infection, 2007, 65:1-8. doi:10.1016/j.jhin.2006.09.026 pmid:17145101

11. Barbour R, Kitzinger J. Developing focus group research: politics, theory and practice. Sage, 1999.

12. Charmaz K. Constructing grounded theory: A practical guide through qualitative analysis. Sage Publications Ltd, 2006.

13. Fewtrell $L$ et al. Water, sanitation, and hygiene interventions to reduce diarrhoea in less developed countries: a systematic review and meta-analysis. The Lancet Infectious Diseases, 2005, 5:42-52. doi:10.1016/S1473-3099(04)01253-8 pmid:15620560

14. Scott B et al. Health in our hands, but not in our heads: understanding hygiene motivation in Ghana. Health Policy and Planning, 2007, 22:225-33. doi:10.1093/heapol/czm016 pmid:17526639

15. Jumaa PA. Hand hygiene: simple and complex. International Journal of Infectious Diseases, 2005, 9:3-14. doi:10.1016/j. ijid.2004.05.005 pmid:15603990 